\title{
Bone Marrow Edema in the Knee and Its Influence on Clinical Outcome After Matrix-Based Autologous Chondrocyte Implantation
}

\section{Results After 3-Year Follow-up}

\author{
Thomas R. Niethammer, ${ }^{*}$ MD, Siegfried Valentin, ${ }^{*}$ MD, Mehmet F. Gülecyüz, ${ }^{*}$ MD, \\ Björn P. Roßbach, ${ }^{\star}$ MD, Andreas Ficklscherer, ${ }^{*}$ MD, Matthias F. Pietschmann, ${ }^{*}$ MD, \\ and Peter E. Müller, ${ }^{\star \dagger}$ MD \\ Investigation performed at the Department of Orthopaedic Surgery, Physical Medicine \\ and Rehabilitation, University Hospital of Munich, Munich, Germany
}

\begin{abstract}
Background: Third-generation autologous chondrocyte implantation (ACl) is an established method for treatment of fullthickness cartilage defects in the knee joint. Subchondral bone marrow edema (BME) is frequently observed after ACl, with unknown pathogenesis and clinical relevance.
\end{abstract}

Purpose: To investigate the occurrence and clinical relevance of BME after third-generation $\mathrm{ACl}$ in the knee joint during the postoperative course of 36 months.

Study Design: Cohort study; Level of evidence, 3.

Methods: A total of 38 circumscribed full-thickness cartilage defects in 30 patients were included in this study. All defects were treated with third-generation $\mathrm{ACl}$ (Novocart 3D). A standardized MRI examination was carried out after 1.5, 3, 6, 12, 24, and 36 months. Bone marrow edema was observed in $78.9 \%$ of defects over the postoperative course, with initial occurrence in the first 12 months. The size of the BMEs were determined according to their maximum diameter and were classified as small $(<1 \mathrm{~cm})$, medium $(<2 \mathrm{~cm})$, large $(<4 \mathrm{~cm})$, and very large (diffuse; $>4 \mathrm{~cm})$. Clinical outcomes in patients were analyzed by use of the International Knee Documentation Committee (IKDC) scoring system and a visual analog scale for pain.

Results: There were 5.3\% ( $n=2)$ small, 28.9\% $(n=11)$ medium, 34.2\% $(n=13)$ large, and 10.5\% $(n=4)$ very large BMEs. In a subgroup analysis, cartilage defects of the medial femoral condyle showed significantly higher frequency of BME than did patellar defects. Clinical scores showed significant improvements throughout the entire study course $(P<.05)$. Clinical patient outcome did not correlate with presence of BME at any time period $(P>.05)$.

Conclusion: Midterm clinical results of the matrix-based third-generation ACl showed a substantial amount of BME over a 36month follow-up, but this did not correlate with worse clinical outcome. Patients with femoral cartilage defects were more often affected than were those with patellar cartilage defects.

Keywords: bone marrow edema; autologous chondrocyte implantation; ACl; cartilage

\footnotetext{
${ }^{\dagger}$ Address correspondence to Peter E. Müller, MD, Department of Orthopaedic Surgery, Physical Medicine and Rehabilitation, University Hospital of Munich (LMU), Marchioninistraße, 15, Munich, 81377, Germany (email: peter.mueller@med.uni-muenchen.de).

*Department of Orthopaedic Surgery, Physical Medicine and Rehabilitation, University Hospital of Munich, Munich, Germany.

T.R.N., A.F., M.F.P., and P.E.M. are members of the working group "Tissue Regeneration" of the German Society for Orthopaedic and Trauma Surgery.

The authors declared that they have no conflicts of interest in the authorship and publication of this contribution.
}

The American Journal of Sports Medicine, Vol. 43, No. 5 DOI: $10.1177 / 0363546515573935$

(C) 2015 The Author(s)
In the past 2 decades, autologous chondrocyte implantation (ACI) has become an established method of treatment of full-thickness cartilage defects of the knee joint. Numerous studies have shown good to excellent clinical results in the medium-term course up to 10 years. ${ }^{1,2,8,20,24}$ Thirdgeneration ACI represents an evolution of the periosteal ACI developed by Brittberg et al. ${ }^{4}$ To this end, chondrocytes are seeded into 3-dimensionally constructed scaffolds for implantation into the cartilage defects. A periosteal flap cover is not required, which simplifies the operative procedure and has comparable midterm results. ${ }^{9}$ Common complications after ACI are symptomatic hypertrophy, 
disturbed fusion, delamination, and graft failure. ${ }^{18,26}$ In addition, subchondral bone marrow edema (BME) has been observed in numerous patients in the postoperative course.

Subchondral BME is an increased signal in the marrow on magnetic resonance imaging (MRI) and can be detected with fat-suppressed T2-weighted sequences. ${ }^{6}$ In clinical practice, BME is often observed after ACI in the knee joint. In previous studies, ${ }^{6,12,15} \mathrm{BME}$ has been associated with cartilage loss and pain. It can be therefore be seen as a potential negative prognostic indicator for cartilage regeneration after third-generation ACI, which could be of fundamental importance for revision surgery.

So far, the incidence and clinical relevance of subchondral BME after third-generation ACI remain unclear. Therefore, a better understanding of the development and occurrence of this condition is necessary to determine potential treatment alternatives. The aim of this study was to investigate the occurrence and frequency of subchondral BME after third-generation ACI at the knee joint. Furthermore, we studied whether subchondral BME has predictive value for the postoperative course of 3 years. We hypothesized that defect-associated subchondral BME was not associated with worse clinical outcomes 3 years after surgery.

\section{METHODS}

\section{Patient Cohort}

For this study, we evaluated all symptomatic cases of knee cartilage defects treated in our department that were classified by 4 different surgeons as grades III and IV according to the International Cartilage Repair Society (ICRS). All defects were treated with third-generation ACI with a collagen/chondroitin sulfate-based biphasic scaffold (Novocart 3D; TETEC GmbH). All patients were treated according to the guidelines of the working group Tissue Regeneration of the German Society for Orthopaedic and Trauma Surgery. ${ }^{17}$

Exclusion criteria for ACI in this study were osteoarthritis of the knee or joint instability, arthritis, corresponding cartilage defects or more than 2 focal cartilage defects, and patients with incomplete data from clinical and MRI analysis. Patients with osteochondritis dissecans and patients with ACI as second-line cartilage treatment (eg, microfracturing) were also excluded from the study.

The dropout rate after 36 months of follow-up was $27.8 \%$; another 32 patients were excluded because of the strict exclusion criteria. A total of 38 consecutive isolated full-thickness cartilage defects in 30 patients $(65.8 \%$ men and $34.2 \%$ women) were included in this study. The mean age of the patients was 36.4 years, and the mean body mass index (BMI) was 26.1. Patient characteristics are described in Table 1.

With institutional review board approval, the cartilage defects were evaluated arthroscopically. Patients were clinically evaluated in a standardized manner preoperatively and after $6,12,24$, and 36 months postoperatively.
TABLE 1

Characteristics of Study Patients $(\mathrm{N}=30)^{a}$

\begin{tabular}{lc}
\hline Age at ACI, y, mean \pm SD (range) & $36.4 \pm 10.8(12-51)$ \\
BMI, mean \pm SD (range) & $26.1 \pm 4.0(19.6-32.2)$ \\
Defect size, $\mathrm{cm}^{2}$, mean $\pm \mathrm{SD}$ (range) & $5.4 \pm 2.3(2-12)$ \\
Sex, $\mathrm{n}(\%)$ & \\
$\quad$ Male & $25(65.8)$ \\
Female & $13(34.2)$ \\
No. of defects & 38 \\
Defect origin, $\mathrm{n}(\%)$ & \\
Acute traumatic & $4(10.5)$ \\
Old traumatic & $17(44.7)$ \\
Degenerative & $17(44.7)$ \\
Defect location, $\mathrm{n}(\%)$ & \\
MFC & $14(36.9)$ \\
LFC & $3(7.9)$ \\
Patella & $17(44.7)$ \\
Trochlear groove & $4(10.5)$
\end{tabular}

${ }^{a} \mathrm{ACI}$, autologous chondrocyte implantation; BMI, body mass index; LFC, lateral femoral condyle; MFC, medial femoral condyle.

Clinical outcomes were analyzed using the International Knee Documentation Committee (IKDC) scoring system and a visual analog scale (VAS) for pain. Results were recorded in the standardized ICRS form.

\section{ACl Procedure}

Two (Novocart 3D) or 3 (Novocart 3D XL) osteochondral plugs were taken from an unloaded area of the intercondylar notch and placed in a sterile nutrient solution. The osteochondral plugs, with a diameter of $3 \mathrm{~mm}$ and a thickness of 5 to $10 \mathrm{~mm}$, were positioned in a transport box and sent to the manufacturer. After a cultivation time of 3 to 4 weeks, the seeded scaffolds were then implanted in a second operation. After a perioperative antibiotic prophylaxis with a single dose of intravenous cefuroxim $1.5 \mathrm{~g}$ (Hikma Pharma), the knee joint was exposed by parapatellar arthrotomy. Depending on the location of the defect site, either medial or lateral arthrotomy was conducted. The cartilage defects were debrided with curettes to obtain a stable rim of healthy surrounding cartilage and were then prepared with punches without damaging the subchondral plate. The sizes of the cartilage defects were measured. The chondrocyte seeded scaffolds were cut with the corresponding punches and inserted into the debrided cartilage defect. Absorbable sutures were used to fix the seeded scaffold in the defect. In 6 patients (15.8\%) without a stable cartilage rim, additional absorbable poly-L-lactide (PLLA) pins (Aesculap AG) were used.

Postoperative rehabilitation was conducted with a standardized protocol as described in the literature. ${ }^{25}$ In patients with femoral cartilage defects, rehabilitation began with use of a continuous passive motion (CPM) device after 24 hours of bed rest and drain removal. Weightbearing was limited to $20 \mathrm{~kg}$ for 6 weeks for femoral cartilage defects, while flexion was increased quickly. In patients with patellar defects, a knee brace was fitted. Flexion with the knee brace was limited to $30^{\circ}$ for 2 to 3 
weeks and was gradually increased in the following weeks. Full weightbearing was allowed with full extension after wound healing. All patients were treated by physical therapists. Moderate physical activities such as cycling, swimming, and Nordic walking were allowed not earlier than 3 months postoperatively. Patients were instructed not to begin playing high-impact sports (eg, soccer, basketball) earlier than 12 months after surgery. ${ }^{22}$

\section{Magnetic Resonance Imaging}

A standardized follow-up MRI examination was carried out after 1.5, 3, and 6 months in the first postoperative year and after 12, 24, and 36 months postoperatively. We used a 1.5-T tomograph with a commercially available circular polarizing 1-channel knee spool (Magnetom Sonata; Siemens). The following sequences were performed: fast-spin echo (dual T2-FSE) and fat-saturation gradient echo (3DGE-FS) and, in addition, proton-weighted sequences and T1-weighted fast-low-angle-shot-sequence (FLASH) with selective water stimulation. BMEs were determined and classified according to their maximum diameter.

The examination and classification of the BMEs were performed by experienced orthopaedic surgeons and radiologists specialized in musculoskeletal radiology. An intraclass correlation coefficient (ICC) analysis was performed. The ICC values showed a "substantial" to "almost perfect" intraobserver reliability (ICC $=0.732-0.834$ ) after the various time points, according to the rating by Landis and Koch. ${ }^{14}$

The BME was measured and evaluated on a postprocessing workstation with Syngo medical imaging software (Siemens Medical Solutions) using DICOM (Digital Imaging and Communications in Medicine) datasets. Classification of the BME, based according to Welsch et $\mathrm{al}^{31}$ on the maximum measured diameter, was as follows: small $(<1 \mathrm{~cm})$, medium $(<2 \mathrm{~cm})$, large $(<4 \mathrm{~cm})$, and very large $(>4 \mathrm{~cm})$.

\section{Statistics}

Statistical analysis was performed by use of SPSS (version 21; IBM Corp). Significant differences between the described follow-up examinations were determined using the Wilcoxon test for dependent samples. The MannWhitney $U$ test was used for independent samples in the comparison of the 2 groups at a certain time period. For comparing the IKDC score after 36 months between the patients with and without BME (Table 2), the group sizes gave 0.75 power to detect a large effect size $(\mathrm{d}=0.91)$ using $t$ test, with alpha at .05 . Post hoc power analysis was performed with G*Power (version 3.1.6). ${ }^{5}$ Results with an error probability of $<5 \%(P<.05)$ were considered significant.

\section{RESULTS}

The treated defects had a mean defect size of $5.4 \mathrm{~cm}^{2}$. Regarding location, $44.8 \%$ of the defects were in the femoral condyle, $44.7 \%$ were in the patella, and $10.5 \%$ were in
TABLE 2

Individual and Defect-Related Factors on BME Occurrence ${ }^{a}$

\begin{tabular}{|c|c|c|c|}
\hline & $\mathrm{BME}$ & No BME & $P$ \\
\hline Sex, n $(\%)$ & & & ns \\
\hline Male & $22(73.3)$ & $3(37.5)$ & \\
\hline Female & $8(26.7)$ & $5(62.5)$ & \\
\hline $\mathrm{BMI}$, mean $\pm \mathrm{SD}$ & $25.4 \pm 4.0$ & $28.4 \pm 3.3$ & $\mathrm{~ns}$ \\
\hline Age, $y$, mean $\pm \mathrm{SD}$ & $35.8 \pm 11.5$ & $38.6 \pm 7.9$ & $\mathrm{~ns}$ \\
\hline Defect size, $\mathrm{cm}^{2}$, mean $\pm \mathrm{SD}$ & $5.2 \pm 2.4$ & $6.0 \pm 1.9$ & ns \\
\hline \multicolumn{4}{|l|}{ Cause, n (\%) } \\
\hline Acute trauma $(<12 \mathrm{mo})$ & $4(13.3)$ & 0 & $\mathrm{~ns}$ \\
\hline Old trauma $(>12 \mathrm{mo})$ & $12(40)$ & $5(62.5)$ & \\
\hline Chronic/degenerative & $14(46.7)$ & $3(37.5)$ & \\
\hline Defect location, n (\%) & & & $.023^{b}$ \\
\hline MFC & $14(46.7)$ & 0 & \\
\hline LFC & $3(10)$ & 0 & \\
\hline Patella & $10(33.3)$ & $7(87.5)$ & \\
\hline Trochlear groove & $3(10)$ & $1(12.5)$ & \\
\hline \multicolumn{4}{|l|}{ IKDC score, mean $\pm \mathrm{SD}$} \\
\hline Preoperative & $41.7 \pm 23.9$ & $36.7 \pm 18.5$ & \\
\hline $6 \mathrm{mo}$ & $52.9 \pm 22.3$ & $45.4 \pm 15.6$ & \\
\hline $12 \mathrm{mo}$ & $58.4 \pm 22.1$ & $52.1 \pm 21.6$ & \\
\hline $24 \mathrm{mo}$ & $57.4 \pm 20.9$ & $47.6 \pm 22.8$ & \\
\hline $36 \mathrm{mo}$ & $57.1 \pm 21$ & $39.1 \pm 18.1$ & \\
\hline
\end{tabular}

${ }^{a}$ There was no relationship between the presence of BME and patient BMI, age, or sex. Regarding defect-related factors, there was a significantly higher rate of femoral BME occurrence; patellar defects had a BME rate of only $61.9 \%(n=13)$. BME, bone marrow edema; BMI, body mass index; IKDC, International Knee Documentation Committee; LFC, lateral femoral condyle; MFC, medial femoral condyle; ns, nonsignificant.

${ }^{b}$ Statistically significant difference between BME presence and defect location $(P<.05)$.

the trochlear groove. The origin of the treated defects is presented in Table 1.

In our patient group, there were $30(78.9 \%)$ BMEs in the postoperative course of 36 months. The BME occurred mostly during the first 6 postoperative months. In 24 defects (63.2\%), the BME developed after 1.5 months, in 3 defects (7.9\%) after 3 months, and in 2 defects (5.3\%) after 6 months. After 12 and 24 months, no new BME was observed. In 1 instance (2.6\%) of a bonding defect of the ACI regenerate, a new BME occurred after 36 months. The prevalence of BME over the observation period was between $52.6 \%$ and $63.2 \%$ (Table 3).

Of the 38 treated cartilage defects only $13.2 \%(n=5)$ showed an early course, with a BME regression up to 6 months. In the majority of defects, a constant BME $(42.1 \% ; \mathrm{n}=16)$ or an intermittent course $(15.8 \% ; \mathrm{n}=6)$ over the entire observation period was observed. A late BME course with an occurrence after 6 months postoperatively was observed in $7.9 \%(n=3)$ (Figure 1$)$.

Surgical revision was performed in 8 patients $(21.1 \%)$ with defect-associated BME. In 6 patients (75\%), the bonding of the regenerative cartilage to the healthy adjacent cartilage was insufficient; this was treated with microfracturing. In 2 of the 8 patients (25\%), revision was necessary because of arthrofibrosis. 
TABLE 3

Incidence and Prevalence of BME $(\mathrm{N}=38 \text { defects })^{a}$

\begin{tabular}{lcccccc}
\hline & \multicolumn{5}{c}{ Follow-up, mo } \\
\cline { 2 - 6 } BME & 1.5 & 3 & 6 & 12 & 24 & 36 \\
\hline New & $24(63.2)$ & $3(7.9)$ & $2(5.3)$ & $0(0)$ & $1(0)$ & $22(57.9)$ \\
Prevalence & $24(63.2)$ & $24(63.2)$ & $20(52.6)$ & $21(55.3)$ & $24(63.2)$ \\
\hline
\end{tabular}

${ }^{a}$ Values are reported as $\mathrm{n}(\%)$. Bone marrow edema (BME) was observed in 30 defects (78.9\%) over the 36-month postoperative follow-up period. The prevalence of BME was $52.6 \%$ to $63.2 \%$ over the entire observation period.

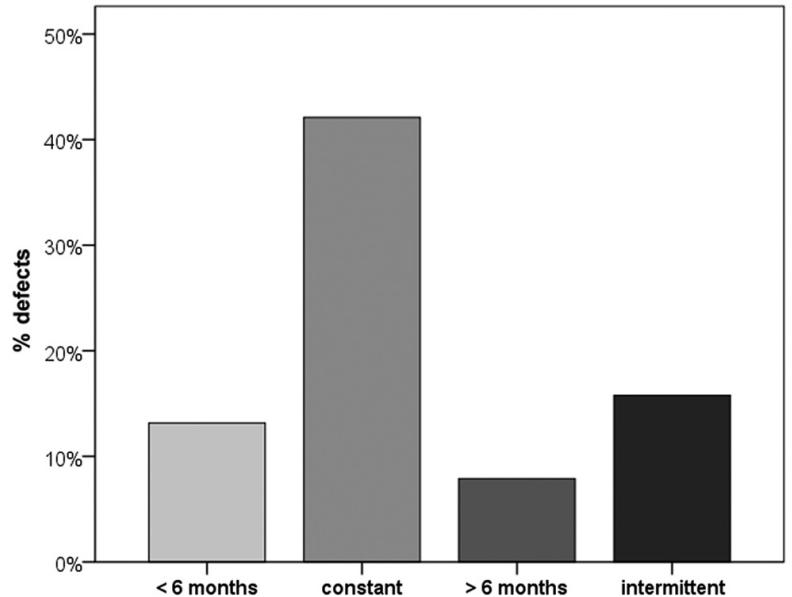

Figure 1. Only $13.2 \%(n=5)$ of the treated cartilage defects showed an early course, with a bone marrow edema regression within 6 months. In the majority of defects, a constant bone marrow edema of $42.1 \%(n=16)$ or an intermittent course of $15.8 \%(n=6)$ over the whole observation period was observed. A late bone marrow edema course with an occurrence after 6 months postoperative was observed in $7.9 \%(n=3)$.

\section{Individual and Defect-Related Factors}

The influence of individual and defect-related factors on the occurrence of BME was also analyzed, but there was no relationship between $\mathrm{BME}$ and patient $\mathrm{BMI}$, age, or sex. Individual and defect-related factors are described in detail in Table 1.

Regarding the defect-related factors, there was a significantly higher rate of femoral BME occurrence. All observed treated femoral defects showed a BME in the postoperative course of 36 months. Patellar defects had a BME rate of only $61.9 \%(n=13)$. The difference observed between BME distribution and defect location was significant $(P=.023)$. Significant differences of the other observed defect-related factors on BME occurrence could not be detected (Table 2).

\section{Correlation Between BME and Clinical Outcomes}

In this study population, we observed a significant increase in the examined clinical scores. There was no difference between the clinical results regarding IKDC score and VAS pain and
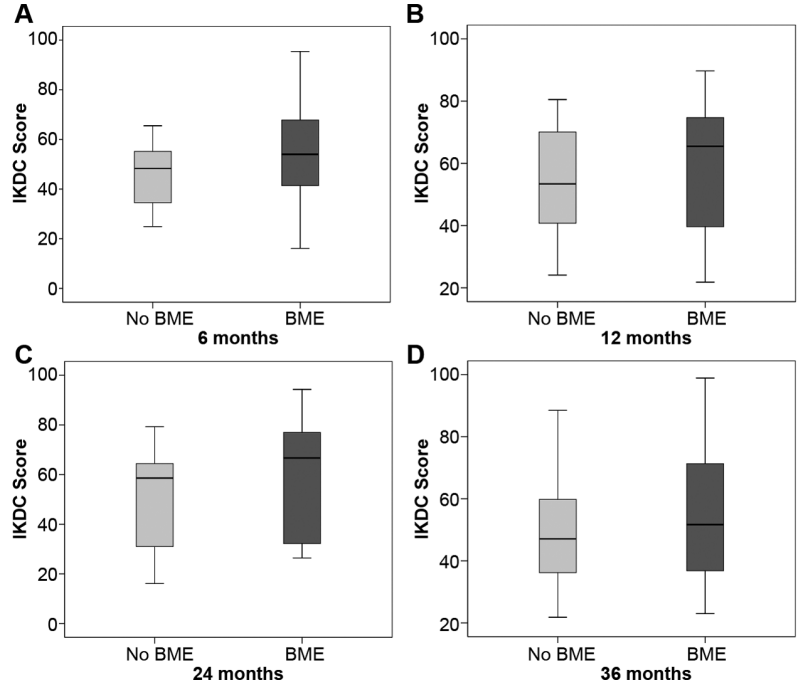

Figure 2. There was no difference between the clinical results regarding the IKDC score and the presence of bone marrow edema during the postoperative follow-up examinations after (A) 6 months, (B) 12 months, (C) 24 months, and (D) 36 months.

the presence of $\mathrm{BME}$ during the postoperative follow-up examinations after 6, 12, 24, and 36 months (Figure 2).

The size of the BME was determined as mentioned in the Materials and Methods section. There were 2 defects $(5.3 \%)$ with a small BME, but in most instances, a medium $(28.9 \%, \mathrm{n}=11)$ or large BME $(34.2 \%, \mathrm{n}=13)$ was observed. Only 4 very large BMEs $(10.5 \%)$ were observed, in only 4 defects $(10.5 \%)$, and only in the first 3 months. No significant differences in the IKDC and VAS scores could be observed in patients with small, medium, large, and very large BMEs or patients without BME. Detailed results of the analyzed clinical scores according to the BME classification are presented in Table 4 . There was no significant difference regarding individual or defect-related factors between the examined BME grades (Figures 3 and 4).

\section{DISCUSSION}

The most important finding of the present study was that there was a high incidence of subchondral BME after third-generation ACI, without worse clinical outcomes. 
TABLE 4

Outcomes Scores at the Various Follow-up Points ${ }^{a}$

\begin{tabular}{|c|c|c|c|c|c|c|}
\hline \multirow[b]{2}{*}{ Follow-up } & \multirow[b]{2}{*}{ No BME } & \multicolumn{4}{|c|}{ BME Size $^{b}$} & \multirow[b]{2}{*}{$P$} \\
\hline & & Small & Medium & Large & Very Large & \\
\hline \multicolumn{7}{|l|}{6 months } \\
\hline Defects, $\%$ & 47.4 & 13.2 & 15.8 & 23.7 & 0 & \\
\hline IKDC score & $45.8 \pm 4.6$ & $56.7 \pm 11.8$ & $60.5 \pm 8.5$ & $52.6 \pm 7.8$ & 0 & .603 \\
\hline \multicolumn{7}{|l|}{ VAS score } \\
\hline During movement & $4.7 \pm 0.8$ & $4.3 \pm 1.8$ & $4.3 \pm 1.5$ & $3.4 \pm 1.1$ & 0 & .807 \\
\hline At rest & $1.2 \pm 0.6$ & $0.8 \pm 0.6$ & $0.5 \pm 0.5$ & $2.3 \pm 1.4$ & 0 & .763 \\
\hline \multicolumn{7}{|l|}{12 months } \\
\hline Defects, $\%$ & 44.7 & 18.4 & 21.1 & 15.8 & 0 & \\
\hline IKDC score & $54.4 \pm 4.6$ & $54.0 \pm 8.1$ & $70.4 \pm 9.6$ & $54.1 \pm 8.5$ & 0 & .304 \\
\hline \multicolumn{7}{|l|}{ VAS score } \\
\hline During movement & $3.0 \pm 0.6$ & $4.7 \pm 1.9$ & $3.3 \pm 1.7$ & $1.5 \pm 0.5$ & 0 & .544 \\
\hline At rest & $0.6 \pm 0.5$ & $0.7 \pm 0.7$ & $0.6 \pm 0.3$ & $0.5 \pm 0.4$ & & .665 \\
\hline \multicolumn{7}{|l|}{24 months } \\
\hline Defects, $\%$ & 42.1 & 18.4 & 18.4 & 21.1 & 0 & \\
\hline IKDC score & $51.8 \pm 6.0$ & $61.1 \pm 8.3$ & $57.8 \pm 15.8$ & $54.0 \pm 8.2$ & 0 & .750 \\
\hline \multicolumn{7}{|l|}{ VAS score } \\
\hline During movement & $4.3 \pm 0.9$ & $2.7 \pm 1.2$ & $4.2 \pm 2.3$ & $3.4 \pm 1.1$ & 0 & .668 \\
\hline At rest & $1.0 \pm 0.4$ & $0.2 \pm 0.1$ & $2.0 \pm 2.0$ & $0.8 \pm 0.3$ & & .431 \\
\hline \multicolumn{7}{|l|}{36 months } \\
\hline Defects, \% & 36.8 & 7.9 & 31.6 & 23.7 & 0 & \\
\hline IKDC score & $48.9 \pm 6.1$ & $55.8 \pm 15.6$ & $52.3 \pm 7.6$ & $60.8 \pm 7.2$ & 0 & .655 \\
\hline \multicolumn{7}{|l|}{ VAS score } \\
\hline During movement & $3.3 \pm 0.7$ & $1.2 \pm 0.8$ & $4.1 \pm 0.9$ & $3.3 \pm 0.9$ & 0 & .613 \\
\hline At rest & $0.7 \pm 0.3$ & $0.6 \pm 0.4$ & $0.9 \pm 0.4$ & $0.9 \pm 0.3$ & & .654 \\
\hline
\end{tabular}

${ }^{a}$ Values are reported as mean \pm SD unless otherwise indicated. There were 2 small bone marrow edemas (BMEs), 11 medium BMEs, and 13 large BMEs. A very large BME was observed in only 4 defects and was only observed in the first 3 months postoperatively. No significant differences were observed in the International Knee Documentation Committee (IKDC) and visual analog scale (VAS) scores in the patients with and without BMEs.

${ }^{b} \mathrm{BME}$ size was graded according to maximum diameter: small $=<1 \mathrm{~cm}$; medium $=<2 \mathrm{~cm}$; large $=<4 \mathrm{~cm} ;$ very large $=>4 \mathrm{~cm}$.

These results confirmed our stated hypothesis, that defectassociated subchondral BME is not associated with worse clinical outcome 3 years postoperatively.

Third-generation ACI is a well-established therapy for full-thickness cartilage defects in the knee joint. Multiple studies have shown a positive effect, with increased function and pain reduction. ${ }^{7,8,13,16,19,23}$ In clinical practice, subchondral BME is a frequent radiological observation in the postoperative course after ACI. In the present study we observed a high overall incidence of BME (78\%) during the postoperative course of 3 years after third-generation ACI. A comparison of first-generation ACI and the present findings shows similar results. Interestingly, a wide range in the incidence of BME is reported in the literature, from $14 \%$ to $88 \% .^{10,20,28-30}$

Regarding third-generation ACI, relatively few studies have analyzed subchondral BME in the postoperative course. In a recent study, Zak et $\mathrm{al}^{32}$ demonstrated significant improvement in clinical scores after third-generation ACI using Novocart 3D, with good clinical and radiological medium-term results. However, the investigators did not report incidence and clinical relevance of subchondral BME.

Niemeyer et $\mathrm{al}^{20}$ found a high rate of defect-associated BME $(78 \%)$ in a study of first-generation ACI with only a single MRI follow-up. But given the absence of a standardized MRI follow-up, the occurrence and development of BME could not be thoroughly evaluated. Comparable results of $\mathrm{BME}$ incidence of $88.4 \%$ were demonstrated by Henderson et $\mathrm{al}^{10}$ after 2 years.

Vasiliadis et $\mathrm{al}^{30}$ found a BME incidence of only $14 \%$ after first-generation ACI in a 1-time follow-up after 9 to 18 years. This small incidence is probably attributable to the late follow-up and absence of data about the postoperative course between 1 and 8 years. Other studies showed a lower BME incidence-47\% $\%^{28}$ or $56 \%^{29}$-at 1 year after first-generation ACI.

In these previously published studies, the first MRI follow-up was conducted after 12 months. To investigate the occurrence and development of subchondral BME during the postoperative course of 3 years, it is necessary to perform periodic MRI examinations, especially in the early postoperative period. Therefore, in this study an initial MRI examination was performed after 6 weeks. After that, MRIs were performed after 3 and 6 months and annually up to 3 years.

We observed that BME is more than a purely postoperative injury of the subchondral plate. Only $13.2 \%$ of defects had an early course of BME development, which vanished within 6 months' follow-up. In particular, the high prevalence of BME in defects- $52.6 \%$ to $63.2 \%$-over the entire 


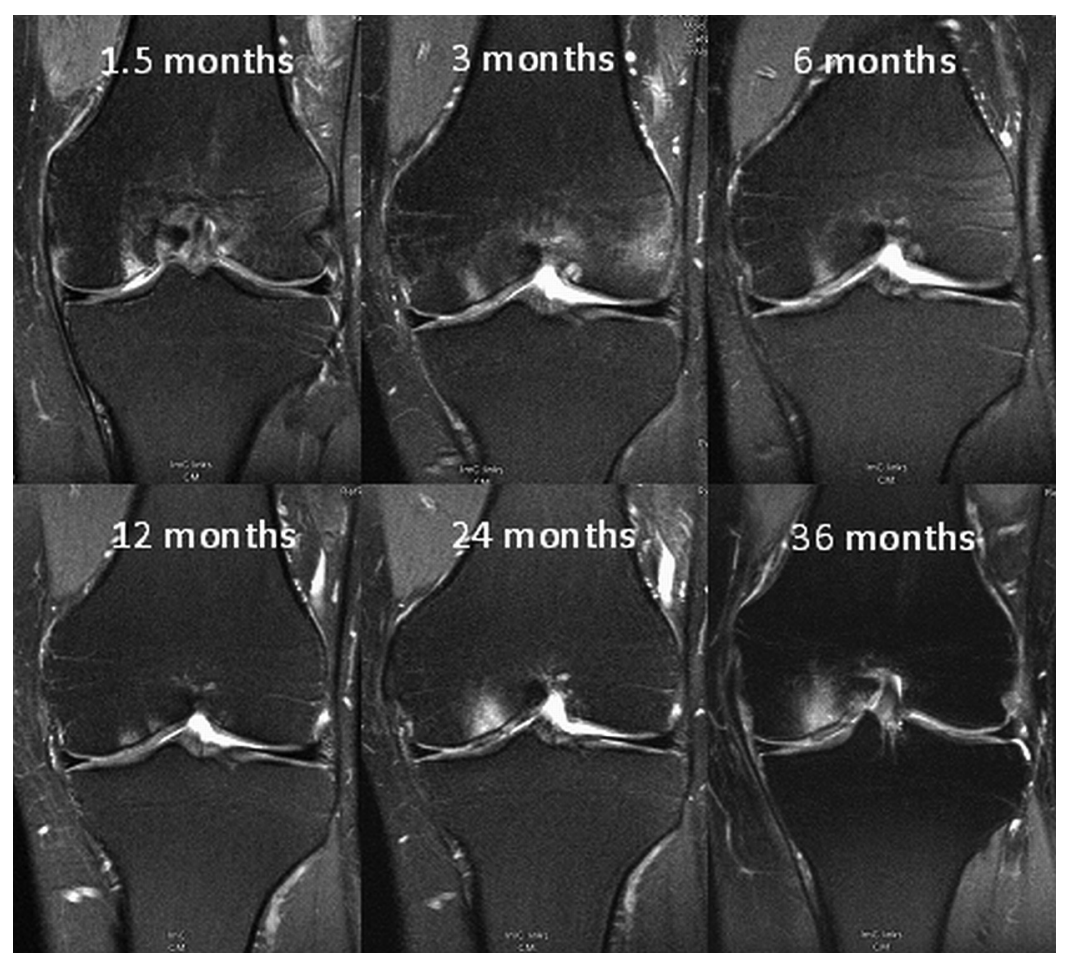

Figure 3. MRI follow-up over 36 months for a 37-year-old man with a treated cartilage defect on the right medial femoral condyle. Defect-associated bone marrow edema could be observed during the entire observation period, but the patient did not report symptoms.

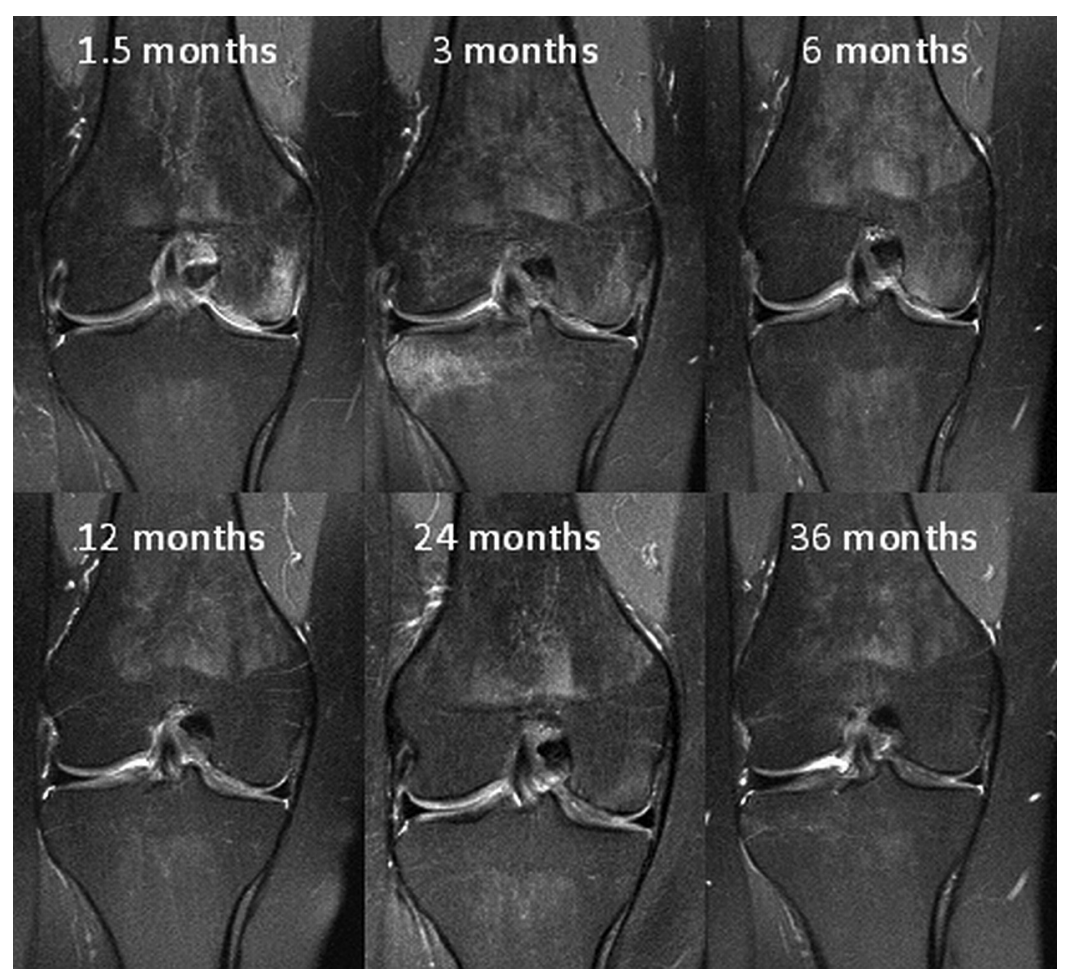

Figure 4. MRI follow-up over 36 months for a 24-year-old woman with a treated cartilage defect on the left medial femoral condyle. Early defect-associated bone marrow edema was observed in the first postoperative months. A non-defect-associated bone marrow edema was observed in the lateral proximal tibia after 3 months. 
observation period in this study suggests that subchondral $\mathrm{BME}$ cannot be seen only as an early postoperative radiological finding.

Also, we found a high rate of late and intermittent occurrence of BME as described above in $23 \%$ of the observed defects. In the majority of cases, a persistent BME could be observed throughout the whole observation period. Graft maturation of ACI cartilage needs at least 12 to 24 months before cartilage regeneration is completed. Therefore, the late or intermittent occurrence of BME can be regarded as a sign of ineffective biomechanical ACI cartilage properties or could perhaps be interpreted as subchondral remodeling with biomechanical adjustment to the changed cartilage properties. Specifically, the latter is suggested by the low improvements in IKDC scores in patients without BME.

Hofmann et $\mathrm{al}^{11}$ described 3 categories of BME: ischemic, mechanical, and reactive. The occurrence of early BME can be interpreted as a postoperative bone marrow edema, which can be seen up to 6 to 12 months. ${ }^{11}$ The occurrence of defect-associated BME is a hypothetical stress-related bone marrow edema caused by mechanical stress. These terms could be seen to indicate a relationship between individual factors or biomechanical changes in cartilage maturation. Therefore, we evaluated possible risk factors in detail in the present study. We observed that all treated femoral defects developed BME during the postoperative course. The occurrence of BME in patellar defects was significantly lower. This finding might be explained by the higher pressure in the femoral condyles compared with retropatellar defects. Niemeyer et al, ${ }^{21}$ analyzing preoperative BMEs and their clinical relevance, detected no relationship between the BME occurrence and defect location.

Vasiliadis et $\mathrm{al}^{30}$ found a higher risk for subchondral cysts with increasing patient age but without relationship to the BME occurrence. Stahl et $\mathrm{al}^{27}$ showed that patient age and BME occurrence could not be associated with osteoarthritis. An influence of the other individual risk factors such as BMI, sex, or defect size could not be detected. These findings were confirmed by Vasiliadis et al. ${ }^{30}$

$\mathrm{BME}$ is frequently observed in patients with symptomatic osteoarthritis of the knee. ${ }^{3}$ Subchondral BME is connected with cartilage defects, as previously described by Kijowski et al. ${ }^{12}$ It can therefore be assumed that the occurrence of subchondral BME is associated with the progression of cartilage degeneration. ${ }^{6}$ Considering these findings, subchondral BME could be a negative prognostic factor for the maturation of the ACI regenerate in the postoperative course. Also, it is questionable whether subchondral BME is a reason for operative revisions in patients with complaints.

Despite numerous clinical studies on prognostic factors, no reliable prognostic factors could be detected until now. Niemeyer et $\mathrm{al}^{21}$ found that a high rate of BME after 12 months is a prognostic factor for a worse clinical outcome after 12 months. The authors stated that the presence of a preoperative subchondral BME may be a reliable prognostic factor for a lower clinical outcome after 12 months. ${ }^{21}$ Nevertheless, a correlation between the presence of a defect-associated BME and the clinical outcome could not be found in the postoperative course. ${ }^{20,30}$ These findings were confirmed with our results. We could not find worse clinical results in the postoperative course of 3 years in patients with a defect-associated BME.

A limitation of the study is the relatively small number of patients and the high dropout rate. A larger study population would helpful for analyzing the subgroups to identify risk factors. In this study population, strict cutoff criteria were defined. Especially, patients with a previous operation (second-line cartilage treatment) with damaging of the subchondral plate (eg, microfracturing) were excluded to eliminate iatrogenic bias. Analysis of the biomechanical properties of the cartilage would have been interesting to look for a relationship between the properties of cartilage and BME occurrence. Preoperative MRI examinations, which were mainly performed outside our clinic, were not included in this study. Arthroscopically performed ACIs and those performed with the use of chondrospheres were not included in our study; therefore, further clinical studies are necessary to show the efficiency and clinical implementation of such procedures.

Finally, the results of the current study show that BME after $\mathrm{ACI}$ in the knee is a common radiological finding in the postoperative course of 3 years. It is still questionable whether defect-associated BME is a negative prognostic factor or even an indication for cartilage degeneration with failed integration into the healthy surrounding cartilage.

In this study, we could not detect worse clinical outcomes in patients with subchondral BME. Due to the high incidence of BME existing in the postoperative period of 3 years, BME is not considered a complication in asymptomatic patients.

\section{CONCLUSION}

This study is the first longitudinal MRI evaluation of defect-associated BME carried out after third-generation ACI in the knee over 3 years. The most important finding of the present study was that there is a high incidence of subchondral BME after third-generation ACI, without worse clinical outcome. With an incidence of 78.9\%, BME is a common radiological phenomenon after ACI, beginning as early as 6 weeks postoperatively. The defect-associated BMEs were observed significantly more frequently in patients with femoral defects. Therefore, it may be theorized that the occurrence of a postoperative defect-associated BME without symptoms could be a sign of a subchondral rebuilding with adjustment to the altered biomechanical function of the articular cartilage in the early prematuration process of the graft cartilage. In cases with symptomatic BME in the postmaturation process in the second postoperative year, other cartilage deficiencies should be excluded.

\section{REFERENCES}

1. Bentley G, Biant LC, Vijayan S, Macmull S, Skinner JA, Carrington RW. Minimum ten-year results of a prospective randomised study of autologous chondrocyte implantation versus mosaicplasty for symptomatic articular cartilage lesions of the knee. J Bone Joint Surg Br. 2012;94(4):504-509. 
2. Beris AE, Lykissas MG, Kostas-Agnantis I, Manoudis GN. Treatment of full-thickness chondral defects of the knee with autologous chondrocyte implantation: a functional evaluation with long-term followup. Am J Sports Med. 2012;40(3):562-567.

3. Brem MH, Schlechtweg PM, Bhagwat J, et al. Longitudinal evaluation of the occurrence of MRI-detectable bone marrow edema in osteoarthritis of the knee. Acta Radiol. 2008;49(9):1031-1037.

4. Brittberg M, Lindahl A, Nilsson A, Ohlsson C, Isaksson O, Peterson L. Treatment of deep cartilage defects in the knee with autologous chondrocyte transplantation. N Engl J Med. 1994;331(14):889-895.

5. Faul F, Erdfelder E, Lang AG, Buchner A. G*Power 3: a flexible statistical power analysis program for the social, behavioral, and biomedical sciences. Behav Res Methods. 2007;39(2):175-191.

6. Felson DT, McLaughlin S, Goggins J, et al. Bone marrow edema and its relation to progression of knee osteoarthritis. Ann Intern Med. 2003;139(5 pt 1):330-336.

7. Filardo G, Kon E, Di Martino A, et al. Second-generation arthroscopic autologous chondrocyte implantation for the treatment of degenerative cartilage lesions. Knee Surg Sports Traumatol Arthrosc. 2012;20(9):1704-1713.

8. Gomoll AH, Gillogly SD, Cole BJ, et al. Autologous chondrocyte implantation in the patella: a multicenter experience. Am J Sports Med. 2014;42(5):1074-1081.

9. Goyal D, Goyal A, Keyhani S, Lee EH, Hui JH. Evidence-based status of second- and third-generation autologous chondrocyte implantation over first generation: a systematic review of level I and II studies. Arthroscopy. 2013;29(11):1872-1878.

10. Henderson I, Francisco R, Oakes B, Cameron J. Autologous chondrocyte implantation for treatment of focal chondral defects of the knee-a clinical, arthroscopic, MRI and histologic evaluation at 2 years. Knee. 2005;12(3):209-216.

11. Hofmann S, Kramer J, Vakil-Adli A, Aigner N, Breitenseher M. Painful bone marrow edema of the knee: differential diagnosis and therapeutic concepts. Orthop Clin North Am. 2004;35(3):321-333, ix.

12. Kijowski R, Stanton $P$, Fine J, De Smet $A$. Subchondral bone marrow edema in patients with degeneration of the articular cartilage of the knee joint. Radiology. 2006;238(3):943-949.

13. Kreuz PC, Muller S, von Keudell A, et al. Influence of sex on the outcome of autologous chondrocyte implantation in chondral defects of the knee. Am J Sports Med. 2013;41(7):1541-1548.

14. Landis JR, Koch GG. The measurement of observer agreement for categorical data. Biometrics. 1977;33(1):159-174.

15. Link TM, Steinbach LS, Ghosh S, et al. Osteoarthritis: MR imaging findings in different stages of disease and correlation with clinical findings. Radiology. 2003;226(2):373-381.

16. Nawaz SZ, Bentley G, Briggs TW, et al. Autologous chondrocyte implantation in the knee: mid-term to long-term results. J Bone Joint Surg Am. 2014;96(10):824-830.

17. Niemeyer $P$, Andereya $S$, Angele $P$, et al. Autologous chondrocyte implantation $(\mathrm{ACl})$ for cartilage defects of the knee: a guideline by the working group "Tissue Regeneration" of the German Society of Orthopaedic Surgery and Traumatology (DGOU). Z Orthop Unfall. 2013;151(1):38-47.

18. Niemeyer P, Pestka JM, Kreuz PC, et al. Characteristic complications after autologous chondrocyte implantation for cartilage defects of the knee joint. Am J Sports Med. 2008;36(11):2091-2099.
19. Niemeyer P, Pestka JM, Salzmann GM, Sudkamp NP, Schmal H. Influence of cell quality on clinical outcome after autologous chondrocyte implantation. Am J Sports Med. 2012;40(3):556-561.

20. Niemeyer $P$, Porichis $S$, Steinwachs $M$, et al. Long-term outcomes after first-generation autologous chondrocyte implantation for cartilage defects of the knee. Am J Sports Med. 2014;42(1):150-157.

21. Niemeyer P, Salzmann G, Steinwachs M, et al. Presence of subchondral bone marrow edema at the time of treatment represents a negative prognostic factor for early outcome after autologous chondrocyte implantation. Arch Orthop Trauma Surg. 2010;130(8): 977-983.

22. Niethammer TR, Muller PE, Safi E, et al. Early resumption of physical activities leads to inferior clinical outcomes after matrix-based autologous chondrocyte implantation in the knee. Knee Surg Sports Traumatol Arthrosc. 2014;22(6):1345-1352.

23. Pestka JM, Bode G, Salzmann G, et al. Clinical outcomes after cell-seeded autologous chondrocyte implantation of the knee: when can success or failure be predicted? Am J Sports Med. 2014;42(1): 208-215.

24. Peterson L, Vasiliadis HS, Brittberg M, Lindahl A. Autologous chondrocyte implantation: a long-term follow-up. Am J Sports Med. 2010;38(6):1117-1124.

25. Pietschmann MF, Horng A, Glaser C, et al. [Post-treatment rehabilitation after autologous chondrocyte implantation: state of the art and recommendations of the clinical tissue regeneration study group of the German Society for Accident Surgery and the German Society for Orthopedics and Orthopedic Surgery.] Unfallchirurg. 2014; 117(3):235-241.

26. Pietschmann MF, Niethammer TR, Horng A, et al. The incidence and clinical relevance of graft hypertrophy after matrix-based autologous chondrocyte implantation. Am J Sports Med. 2012;40(1):68-74.

27. Stahl R, Jain SK, Lutz J, et al. Osteoarthritis of the knee at 3.0 T: comparison of a quantitative and a semi-quantitative score for the assessment of the extent of cartilage lesion and bone marrow edema pattern in a 24-month longitudinal study. Skeletal Radiol. 2011; 40(10):1315-1327.

28. Takahashi T, Tins B, McCall IW, Richardson JB, Takagi K, Ashton K. MR appearance of autologous chondrocyte implantation in the knee: correlation with the knee features and clinical outcome. Skeletal Radiol. 2006;35(1):16-26.

29. Tins BJ, McCall IW, Takahashi T, et al. Autologous chondrocyte implantation in knee joint: MR imaging and histologic features at 1-year follow-up. Radiology. 2005;234(2):501-508.

30. Vasiliadis HS, Danielson B, Ljungberg M, McKeon B, Lindahl A, Peterson L. Autologous chondrocyte implantation in cartilage lesions of the knee: long-term evaluation with magnetic resonance imaging and delayed gadolinium-enhanced magnetic resonance imaging technique. Am J Sports Med. 2010;38(5):943-949.

31. Welsch GH, Zak L, Mamisch TC, Resinger C, Marlovits S, Trattnig S. Three-dimensional magnetic resonance observation of cartilage repair tissue (MOCART) score assessed with an isotropic threedimensional true fast imaging with steady-state precession sequence at 3.0 Tesla. Invest Radiol. 2009;44(9):603-612.

32. Zak L, Albrecht C, Wondrasch B, et al. Results 2 years after matrixassociated autologous chondrocyte transplantation using the Novocart 3D Scaffold: an analysis of clinical and radiological data. Am J Sports Med. 2014;42(7):1618-1627.

For reprints and permission queries, please visit SAGE's Web site at http://www.sagepub.com/journalsPermissions.nav 